\title{
Determine Frequency Adjustment Reserve Based on AGC Regulation Capacity Demand's Random Fuzzy Model
}

\author{
Liqiang PAN ${ }^{1}$, Zhixuan ZHU 2,a , Haifeng YU ${ }^{3}$, Leixin LUO $^{3}$, Xing JIANG ${ }^{3}$ and Zhenyu WU² \\ ${ }^{1}$ State Grid Hunan Electric Power Company Limited, Changsha 410000, China \\ ${ }^{2}$ Changsha University of Science \& Technology, Changsha 410000, China \\ ${ }^{3}$ State Grid Hunan Electric Power Company Economic and Technological Research Institute, Changsha 410004, China
}

\begin{abstract}
The uncertainty of wind power has a negative impact on the frequency adjustment of the system, so the frequency fluctuation caused by wind power can be suppressed only when the system has sufficient frequency adjustment reserve. Only considering randomness or fuzziness cannot describe the uncertainty of wind power, and it is impossible to determine the frequency adjustment requirement of the system. The random fuzzy uncertainty model of AGC regulating capacity demand is proposed, established and verified in this paper, And based on the model, the frequency adjustment reserve can be detemined.
\end{abstract}

\section{Introduction}

In recent years, China's wind power has developed rapidly. By 2017, the installed capacity of wind power in China (except Hong Kong, Macao and Taiwan) was 19.66 million kilowatts, with an accumulative installed capacity of 188 million kilowatts, an increase of $11.7 \%$ over the same period of last year ${ }^{[1]}$. The uncertainties of wind power output will have an negligible impact on the frequency stability of the system, Therefore, maintaining frequency stability is of great significance to wind power absorption, and automatic generation control (AGC), as one of the important means of frequency modulation, has become a research hotspot.

Adequate frequency modulation reserve can ensure the frequency stability of the system when it is disturbed. Due to the wind power access, the frequency adjustment reserve put forward higher requirements. Reference[2] quantitatively analyses the impact of wind power access on the system frequency adjustment system from the indicators of frequency deviation, frequency adjustment speed and frequency modulation capacity. It is concluded that the secondary frequency adjustment reserve needs to be increased under high proportion wind power, but the method to determine the secondary frequency adjustment reserve under wind power integration is not given. Reference [3] introduces that wind turbines participate in frequency adjustment through analogy inertia control, droop control, rotor speed control, pitch angle control and coordinated control strategy, that is, wind farms have a certain frequency adjustment reserve to ensure the ability to participate in frequency adjustment under various circumstances, so as to reduce the impact of wind power on frequency. Reference [7] puts forward a random fuzzy uncertainty model of daily wind speed, analyses the characteristics of probability distribution of daily wind speed and the fuzziness of probability distribution parameters, and the simulation results show that it can be effectively used for daily wind speed simulation. It can be seen from the current research that there is no quantitative analysis of the impact of wind power output on frequency adjustment reserve and the method to determine frequency adjustment reserve.

Considering the uncertainty of wind power, this paper presents and verifies the random fuzzy model of AGC adjustment capacity demand, which is suitable for the optimization of system frequency adjustment reserve under the access of distributed generation.

\section{AGC Adjusting Capacity Demand Random Fuzzy Model}

Uncertainty includes randomness and fuzziness, which cannot be confused. The random fuzzy model of AGC adjusted capacity demand is established by the analysis of randomness and fuzziness. Therefore, the AGC adjustment capacity demand of the random fuzzy model to accurately reflect the impact of wind power uncertainty on the regulation of capacity requirements.

\subsection{Random characteristics analysis and probability model of AGC regulating capacity demand}

Wind power output is random affected by natural law, so the probability statistical analysis is carried out according

a Corresponding author: 313195930@qq.com 
to the randomness of AGC regulation capacity demand. The probability density function is obtained by extracting the distribution characteristics of the regulation capacity demand of $\mathrm{AGC}$, and then the difference of the distribution characteristics of the probability density function is analysed to calculate the probability distribution parameters of the regulation capacity demand of AGC, which lays a foundation for the fuzziness analysis of the following parameters. First, a random analysis of the AGC adjusted capacity requirement is made, and an AGC adjustment capacity demand probability density curve is produced in units of days. According to the characteristics of the AGC adjustment capacity demand probability density change trend, the probability density curve is fitted and the fitting accuracy is verified by the fitting coefficient ( $\mathrm{R}$-Square).

The probability density curve of AGC adjustment capacity demand is obtained every day in a time interval (such as a quarter), but each probability density curve is not exactly the same, so the parameters of the probability density fitting function and the fitting result will not be the same. Therefore, the random model of AGC adjustment capacity demand in a quarter cannot be regarded as a parameter-determined distribution function, and its distribution parameters are ambiguous and should be analysed for fuzziness.

\subsection{Fuzzy Analysis of AGC Adjusting Capacity Demand Probability Distribution Parameters}

Based on the analysis of random characteristics, each AGC adjustment capacity demand probability density curve is not exactly the same, so the parameters of the probability density function fitting function in a quarter need to be represented by the fuzzy parameter formal function in the uncertainty theory. By fitting the probability density curve of AGC adjustment capacity demand in a quarter, the probability distribution of $n$ groups of parameters is obtained (n depends on the result of the fitting function), and the regions in which each parameter is concentrated are statistically analysed, and the parameters are obtained. The frequency distribution is represented and the parameters are represented by fuzzy variables. In general, the fuzzy variables of the parameters can be represented in a fuzzy form according to the frequency distribution of the parameters. The common fuzzy forms are triangular fuzzy numbers and trapezoidal fuzzy numbers. As shown in Figure 1, the characteristic of triangular fuzzy variables is that the frequency distribution of the parameters is the largest at a certain value, and the farther the central value is in the range of occurrence, the occurrence The smaller the frequency, the frequency distribution is similar to a triangle, then this parameter can use the triangle fuzzy variable to describe the distribution parameter; the trapezoidal fuzzy variable is characterized by the fact that the frequency of the parameter is more concentrated in a certain range and away from the concentration The farther the range is, the smaller the frequency is, and the frequency distribution is similar to the trapezoid. This parameter can use the trapezoidal fuzzy variable to describe the distribution parameter.
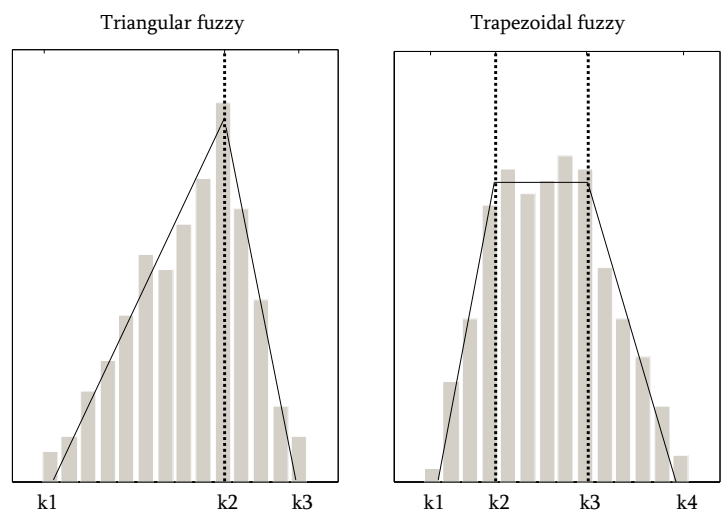

Figure 1. The form of fuzzy variables

Statistical analysis of the parameters of the fitting function results in a quarter (calculating the confidence level of each parameter has a $95 \%$ confidence interval), and taking the minimum value of the confidence interval as the lower limit of the fuzzy number parameter of the time interval, taking the maximum value of the confidence interval As the upper limit of the fuzzy number parameter of the time distribution, the central value of the frequency of occurrence of the parameter needs to be determined for the triangular fuzzy variable, and the central region of the frequency of occurrence of the parameter is determined for the trapezoidal fuzzy variable, thereby obtaining the membership function of the parameter. Where the triangular fuzzy membership function can be expressed as: $\gamma=\left(k_{1}, k_{2}, k_{3}\right)$, the trapezoidal fuzzy membership function can be expressed as: $\gamma=\left(k_{1}, k_{2}, k_{3}, k_{4}\right)$,The specific forms of expression are:

$$
\begin{gathered}
\gamma=\left\{\begin{array}{lr}
0, & x \leq k_{1} \\
\frac{x-k_{1}}{k_{2}-k_{1}}, & k_{1}<x \leq k_{2} \\
\frac{k_{3}-x}{k_{3}-k_{2}}, & k_{2}<x \leq k_{3} \\
0, & x>k_{3}
\end{array}\right. \\
\gamma=\left\{\begin{array}{lr}
0, & x \leq k_{1} \\
\frac{x-k_{1}}{k_{2}-k_{1}}, & k_{1}<x \leq k_{2} \\
1, & k_{2}<x \leq k_{3} \\
\frac{k_{4}-x}{k_{4}-k_{3}}, & k_{3}<x \leq k_{4} \\
0, & x>k_{4}
\end{array}\right.
\end{gathered}
$$

\section{AGC Adjusted Capacity Demand Random Fuzzy Simulation}

AGC adjustment capacity requirement refers to the automatic generation control system according to the 
current frequency deviation to control the units' output to restore frequency stability. In dealing with the problem of wind power, the wind power connected to the system is equivalent to a negative load. The net load is obtained by adding the original load and the wind load.

$$
P_{N}(t)=P_{L}(t)+P_{W}(t)
$$

Where $\mathrm{P}_{\mathrm{N}}$ is the net load; $\mathrm{P}_{\mathrm{L}}$ is the original load; $\mathrm{P}_{\mathrm{W}}$ is the wind power load.

The random fuzzy simulation of AGC adjustment capacity demand is divided into two parts: uncertain feature extraction and regulation capacity demand simulation. The specific procedures are as follows:

1) First, according to the net load data, we get the daily AGC adjustment capacity requirement and plot the probability density curve, then perform a function fitting on the probability density curve;

2) After fitting the daily AGC adjusted capacity demand probability density curve in a quarter, the parameters of the fitting curve are arranged, and the fuzzy form of the parameter is determined, and the probability density curve of the AGC adjusting capacity demand containing the fuzzy parameter is determined;

3) Extracting the random number from the membership function to which the fuzzy parameter belongs and introducing it into the formula containing the probability density curve of the fuzzy parameter, and inversely transforming the formula to obtain the simulation curve of AGC adjusting capacity demand;

4) Verify the correctness of the model and determine the range of adjustment capacity requirements through the AGC adjustment of the capacity demand simulation curve to determine the frequency adjustment reserve.

\section{Determination the frequency adjustme nt reserve}

According to the previous section, the AGC adjusts the random fuzzy model of capacity demand. This section verifies the validity of the model by example.

Taking a city from April to June 2016 as an example, a random fuzzy model of AGC adjusted capacity demand was established and verified. Firstly, the probability density curve of AGC adjusted capacity demand in a quarter is plotted and the function is fitted. The statistical results show that the AGC adjusted capacity demand probability density trend shows a simple unimodal characteristic. The fitting results are shown in the following example:

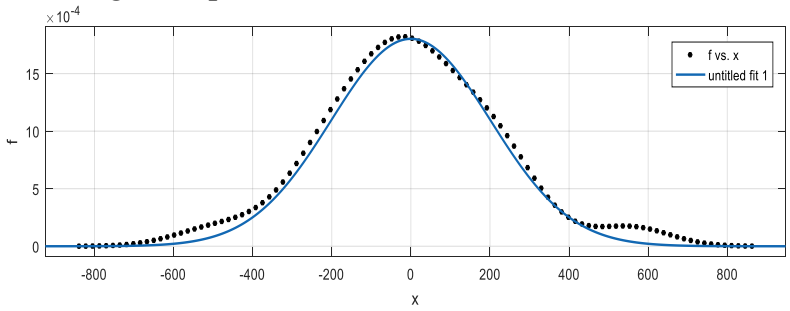

Figure 2. Probability density curve fitting of AGC regulation capacity requirements
According to the daily AGC adjustment of the distribution probability density distribution, the curve fitting tool is used to fit the probability density distribution result. In this example, the Gaussian distribution function is used, as shown in equation (4), and the fitting curve and the original The probability density curve is the closest and the highest fit.

$$
f\left(S_{A G C}\right)=a e^{-\left(\frac{S_{A G C}-b}{c}\right)^{2}}
$$

Where $\mathrm{S}_{\mathrm{AGC}}$ is the $\mathrm{AGC}$ to adjust capacity requirements, and $\mathrm{a}, \mathrm{b}$, and $\mathrm{c}$ are probability density parameters.

According to the fitting result of R-Square, the closer the R-Square is to 1 , the better the fitting effect of the fitting function and the original function. The daily fitting coefficient of April-June 2016 is as shown in the following table:

Table 1. The result of probability density curve fitting

\begin{tabular}{cccccccc}
\hline April & 0.987 & 0.998 & 0.987 & 0.992 & 0.993 & 0.985 & 0.986 \\
\hline 0.989 & 0.992 & 0.988 & 0.985 & 0.993 & 0.998 & 0.984 & 0.992 \\
\hline 0.973 & 0.994 & 0.988 & 0.980 & 0.983 & 0.984 & 0.981 & 0.983 \\
\hline 0.986 & 0.988 & 0.992 & 0.984 & 0.981 & 0.993 & 0.985 & \\
\hline May & 0.985 & 0.982 & 0.984 & 0.981 & 0.981 & 0.989 & 0.982 \\
\hline 0.993 & 0.995 & 0.996 & 0.997 & 0.991 & 0.988 & 0.981 & 0.984 \\
\hline 0.975 & 0.986 & 0.986 & 0.991 & 0.994 & 0.993 & 0.991 & 0.982 \\
\hline 0.981 & 0.995 & 0.988 & 0.993 & 0.996 & 0.996 & 0.984 & 0.991 \\
\hline June & 0.983 & 0.994 & 0.995 & 0.998 & 0.997 & 0.998 & 0.992 \\
\hline 0.998 & 0.993 & 0.996 & 0.995 & 0.985 & 0.964 & 0.990 & 0.990 \\
\hline 0.993 & 0.982 & 0.993 & 0.985 & 0.998 & 0.999 & 0.997 & 0.995 \\
\hline 0.990 & 0.991 & 0.993 & 0.986 & 0.986 & 0.988 & 0.993 & \\
\hline
\end{tabular}

As shown in the above table, the fitting coefficient is about 0.98, which shows that the fitting result is good. Next, the parameters in the simulation and results are fuzzified to obtain the probability density function and determine the fuzzy form of the parameters. The frequency distribution of the parameters is as follows:
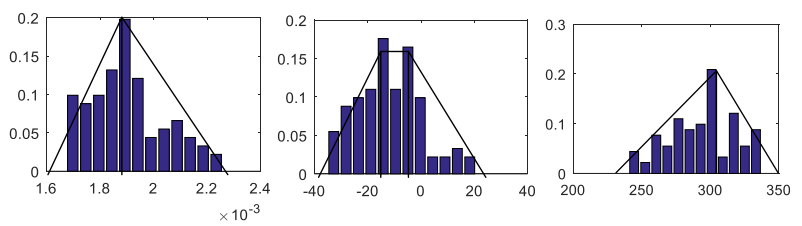

Figure 3. Parameter probability distribution diagram

According to Figure 3, the parameters a and c belong to triangular fuzzy form, and the parameters $b$ belong to trapezoidal fuzzy form. Then the membership functions of the three parameters are obtained. Based on (5)determine the AGC capacity requirement of the system:

$$
S_{A G C}=b+c\left(\ln \frac{a}{f\left(S_{A G C}\right)}\right)^{1 / 2}
$$

The result of adjusting the capacity requirement is shown in the following figure. The simulation results show that the probability of the AGC adjustment capacity requirement in the corresponding upper and lower limits 
of the case data is more than $94 \%$, which indicates that the proposed model can predict AGC accurately and effectively.

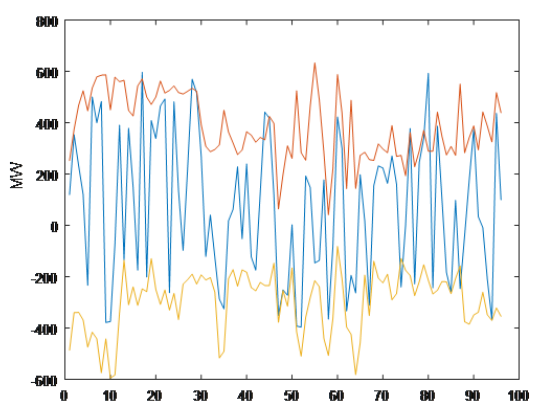

Figure 4. Random fuzzy simulation results of AGC regulation demand

When the unit output can track the AGC regulation demand, the frequency of the system can be maintained stable, so the predicted maximum of the AGC regulation capacity demand is used as the frequency modulation reserve of the system.

\section{Conclusions}

Wind power access brings difficulty to system frequency adjustment. Because of the uncertainty of wind power, it is difficult to estimate the required frequency adjustment reserve. Uncertainty includes randomness and fuzziness. Only considering randomness or fuzziness cannot determine the adjustment capacity demand. The main results and conclusions are as follows:

1) A random fuzzy model of AGC adjustment capacity requirement considering wind power uncertainty is established and its rationality and validity are verified.

2) Determine the reserve capacity of AGC according to the maximum AGC adjusted demand.

\section{References}

1. 2017 China Wind Power Installed Capacity Statistics Briefing [R]. (2018)

2. DING Li, QIAO Ying, LU Zongxiang, et al. Impact on Frequency Regulation of Power System from Wind Power with High Penetration[J]. Automation of Electric Power Systems, 38(14): 1-7 (2014)

3. LIU Ju, YAO Wei, WEN Jinyu, et al. Prospect of Technology for Large-Sc ale Wind Farm Participating Into Power Grid Frequency Regulation [J]. Power System Technology, 38(3): 638 -645 (2014)

4. Kassaei H.R, Keyhani A, Woung T, et al. Ahybrid fuzzy, neural network bus load modeling and predication[J]. IEEE Transactions on Power Systems, 14(2): 718-724 (1999)

5. Nikolay Nikolaev, Yulian Rangelov, Konstantin Gerasimov. Influence of wind turbine generators on the frequency of the electric power system during large disturbances $[\mathrm{J}]$. 12th International Conference on Environment and Electrical Engineering (2013)
6. Davide Fabozzi, Nina F. Thornhill, Bikash C. Pal.Frequency restoration reserve control scheme with participation of industrial loads [J]. IEEE Grenoble Conference (2013)

7. MA Rui, ZHANG Qiang, WU XIA, et al. Random Fuzzy Uncertain Model for Daily Wind Speed[J]. Proceedings of the CSEE, 35(24): 6351-6358 (2015)

8. AnduDukpa, B. Venkatesh, Liuchen Chang, Fuzz Stochastic Programming Method: Capacitor Planning in Distribution Systems With Wind Generators[J]. IEEE Transactions on Power Systems, 26(4): 1971-1979 (2011) 